\title{
PLANEJAMENTO ESTRATÉGICO SITUACIONAL: ESTUDO DE CASO EM UMA FARMÁCIA BÁSICA MUNICIPAL
}

\section{SITUACIONAL STRATEGIC PLANNING: A CASE STUDY IN A CITY PRIMARY PHARMACY}

\section{Priscila Lima de Araujo SCALERCIO'; Alexandra Ingrid dos Santos CZEPULA²}

1 - Departamento de Farmácia, Programa de Pós-Graduação em Ciências Farmacêuticas, Universidade Federal do Paraná (UFPR), Curitiba, PR, Brasil

2 - Farm. B. Msc. PhD. / Faculdades Pequeno Príncipe, Curitiba/PR

\section{RESUMO:}

Esse artigo apresenta o Planejamento Estratégico Situacional de Carlos Matus, como mecanismo transformador de realidade dentro da gestão da Assistência Farmacêutica em um município do estado do Paraná. O objetivo deste trabalho foi de analisar a aplicação do método em uma farmácia básica a fim de melhorar a qualidade no atendimento ao usuário. O problema priorizado resultante foi a pouca qualidade no atendimento aos usuários. Sua exploração revelou como causa convergente a ausência de um departamento de Assistência Farmacêutica no município. Esse resultado evidencia a importância da Assistência Farmacêutica para a prestação dos serviços aos usuários do Sistema Único de Saúde que, apesar de regulamentada dentro do sistema, ainda apresenta desafios a sua implantação integral.

Palavras-chave: Planejamento Estratégico Situacional; Gestão em Saúde; Assistência Farmacêutica.

\section{ABSTRACT:}

This article presents the Situational Strategic Planning method of Carlos Matus, as reality transformer mechanism within the management of the Pharmaceutical Care in a state of Paraná County. The objective of this study was to analyze the application of the method in a basic pharmacy in order to improve the quality of care to the user. The low quality of care users was prioritized problem. His exploration revealed as a cause greater the absence of pharmaceutical care in the county. These findings highlight the importance of pharmaceutical care for the provision of services to users of the Unified Health System that, although regulated within the system also presents challenges for its full implementation.

Keywords: Situational Strategic Planning; Health Management; Pharmaceutical Services.

\section{INTRODUÇÃO}

A assistência terapêutica integral, incluindo Assistência Farmacêutica (AF), está inserida na política do Sistema Único de Saúde (SUS) (BRASIL, 1990). A prestação da AF está legitimada nos princípios do sistema, a universalidade, igualdade e integralidade. 
(VIEIRA, 2010). E sua organização deve ser baseada nos princípios da descentralização político-administrativa, hierarquização, e regionalização. (BRASIL, 1998).

A AF dentro do SUS, através da Política Nacional de Medicamentos de 1988, foi entendida como atividades relacionadas ao medicamento, destinadas a sustentar as ações em saúde, um conjunto de práticas voltadas para a promoção, prevenção e recuperação de saúde. Incluindo principalmente as ações relacionadas ao abastecimento de medicamentos com segurança e eficácia, a fim de promover o seu uso racional (BARRETO; GUIMARÃES, 2010). Em 2004, com a Política Nacional da Assistência Farmacêutica, a AF tem seu conceito ampliado além do foco do abastecimento. A nova política adicionou a importância da atenção farmacêutica como prática norteadora das atividades do farmacêutico dentro da AF. (BRASIL, 2004) Juntando as contribuições dessas duas políticas, a AF atende a necessidade do SUS de humanização do atendimento, estabelecimento de vínculo e acolhimento em relação ao usuário. (OLIVEIRA; ASSIS; BARBONI, 2010).

O acesso da população ao sistema de saúde público provocou mudanças contínuas na organização da AF, de maneira a aumentar a cobertura da distribuição gratuita de medicamentos e ao mesmo tempo minimizar custos, além da construção de um modelo de funcionamento que garantisse a descentralização das ações. (OLIVEIRA; ASSIS; BARBONI, 2010).

Atualmente, pode-se perceber a AF consolidada em algumas estruturas de organização e financiamento, entretanto problemas operacionais são verificados não garantindo o seu cumprimento adequado. Problemas como baixa disponibilidade e descontinuidade de medicamentos, dispensação por trabalhadores não qualificados, condições de armazenamento não adequadas ainda são verificados no sistema. (BARRETO; GUIMARÃES, 2010).

Para que políticas públicas, como a AF, sejam implantadas integralmente é preciso que sejam assegurados os recursos necessários à execução das atividades e também que sua gestão seja eficaz, efetiva e eficiente. (OLIVEIRA; ASSIS; BARBONI, 2010).

O planejamento, como uma ferramenta de trabalho, assume papel vital no direcionamento das ações, assim pode-se incluir as políticas públicas. (VIEIRA, 2009).

Um sistema, como o de saúde, sem planejamento, se subjetiva, se fragmenta, e se desorganiza. Segundo Vieira, 2009, no planejamento do SUS há iniciativas e avanços decorrentes de modo à integração e ordenação, mas ainda apresentam-se obstáculos e vazios a essa finalidade. 
O planejamento estratégico situacional (PES) é uma ferramenta de planejamento alternativa para modelos complexos, como o sistema público. Esse método, ao contrário do planejamento tradicional, exige um correto entendimento e interpretação do cenário do problema a ser solucionado. Consiste no fato de não entender um cenário como isolado e estático, não separar as funções de planejamento e execução, e realizar análises situacionais para orientar as ações, devendo a realidade ser constantemente acompanhada. (IIDA, 1993).

Sendo assim, esse trabalho teve como objetivo analisar a aplicação do método PES em uma farmácia básica.

\section{MÉTODOS}

Trata-se de um relato de experiência do tipo estudo de caso dos resultados obtidos do desenvolvimento de um plano operativo por meio do PES. O foco do plano operativo foi uma das cinco farmácias básicas municipais que compreendem o serviço AF de um município de médio porte do Paraná. Participaram do planejamento, todos os funcionários da secretaria municipal de saúde, relacionados ao foco, que se interessaram e concordaram com suas participações através do termo de consentimento livre e esclarecido.

A metodologia PES desenvolvida pelo economista chileno Carlos Matus (IIDA, 1993; MATUS, 1993), é composto por quatro etapas:

1) Momento explicativo: fase de identificação de problemas a partir da perspectiva dos diferentes atores relacionados, refere-se a um diagnóstico situacional. Como resultado desse momento tem-se a priorização dos problemas pelos atores.

2) Momento normativo: corresponde ao desenho da realidade que se almeja através da definição de objetivos específicos, incluindo o a definição das etapas necessárias a esse resultado.

3) Momento estratégico: momento de análise das ações e operações do planejamento, verificando sua viabilidade e elaborando estratégias a fim de contornar empecilhos.

4) Momento tático operacional: é o momento da ação acompanhado da formulação de indicadores de avaliação e de monitoramento da execução.

A condução das oficinas levou em consideração o entendimento dinâmico da ferramenta, contemplando a interações entre os momentos e a reavaliações constantes. 


\section{RESULTADOS}

O plano operativo foi desenvolvido através da realização de oficinas periódicas. No primeiro momento, ocorreu a identificação dos problemas pelos atores, conforme tabela 1.

\section{TABELA 1 - PROBLEMAS IDENTIFICADOS PELOS ATORES}

\section{PROBLEMAS IDENTFICADOS}

Falta de Medicamentos;

Ausência de um sistema informatizado que atenda as demandas de gerenciamento, controle e atendimento da farmácia;

Pouca qualidade no atendimento aos usuários;

Não atendimento exato a quantidade de medicamentos prescritos na receita;

Farmácia vinculada a Unidade Básica de Saúde, porém localizadas a distância;

Falha de comunicação interna entre a farmácia e os profissionais da Unidade Básica de Saúde;

Falta de recursos humanos qualificados e em quantidade suficiente;

Infraestrutura inadequada;

Farmácia vinculada a Unidade Básica de Saúde, porém com característica de atendimento regional;

Inexistência de protocolos de dispensação e prescrição;

Inexistência de um setor de Assistência Farmacêutica no município;

Desconhecimento da Relação Municipal de Medicamentos (REMUME) pelos profissionais prescritores.

Os problemas coletados foram classificados através de uma matriz de priorização. A matriz de priorização atribuiu valores aos problemas de acordo com avaliação de atributos de magnitude, transcendência, vulnerabilidade, urgência e factibilidade. Como resultante a aplicação da matriz, o problema: a pouca qualidade no atendimento aos usuários obteve maior pontuação, sendo o problema priorizado nesse trabalho.

A insatisfação com o serviço; a baixa adesão ao tratamento; e a utilização incorreta 
de medicamentos foram apontados como evidências da existência do problema. Concluindo o momento explicativo, o problema foi analisado através do apontamento de causas e consequências. O resultado obtido foi contemplado através de um diagrama de Ishikawa ou matriz de espinha de peixe. A aplicação da matriz permitiu a visualização da amplitude do cenário do problema priorizado, através da indicação de uma causa e consequência convergente.

A partir da causa convergente, a ausência de uma coordenação farmacêutica para o serviço, e da consequência convergente, o mau uso dos recursos definiu-se o objetivo geral de promover a implantação de um modelo de Assistência Farmacêutica no município. As causas e consequências secundárias foram definidores dos objetivos específicos para os quais foram estabelecidas as operações e ações, conforme matriz do momento normativo descrita na TABELA 2.

TABELA 2 - MATRIZ DO MOMENTO NORMATIVO

\begin{tabular}{|c|c|c|}
\hline OBJETIVOS ESPECÍFICOS & OPERAÇÕES & AÇÕES \\
\hline \multirow{8}{*}{$\begin{array}{l}\text { Promover a implantação de } \\
\text { uma coordenação } \\
\text { farmacêutica para o serviço, } \\
\text { a fim de melhorar o uso dos } \\
\text { recursos empregados. }\end{array}$} & \multirow{3}{*}{$\begin{array}{c}\text { Elaboração e aprovação } \\
\text { de um projeto de } \\
\text { coordenação } \\
\text { farmacêutica para o } \\
\text { serviço. }\end{array}$} & $\begin{array}{l}\text { Reunir os farmacêuticos do } \\
\text { município interessados. }\end{array}$ \\
\hline & & $\begin{array}{l}\text { Elaborar o projeto de coordenação } \\
\text { farmacêutica para o serviço. }\end{array}$ \\
\hline & & $\begin{array}{c}\text { Apresentar o projeto e solicitar } \\
\text { aprovação dos setores relacionados. }\end{array}$ \\
\hline & \multirow{4}{*}{$\begin{array}{c}\text { Promoção sobre a } \\
\text { importância da } \\
\text { implantação de uma } \\
\text { coordenação } \\
\text { farmacêutica junto ao } \\
\text { meio de controle social. }\end{array}$} & $\begin{array}{c}\text { Obter participação no conselho de } \\
\text { saúde municipal como representante } \\
\text { da categoria farmacêutica. }\end{array}$ \\
\hline & & $\begin{array}{c}\text { Defender a importância } \\
\text { organizacional de uma coordenação } \\
\text { farmacêutica. }\end{array}$ \\
\hline & & $\begin{array}{l}\text { Verificar a deficiência entre } \\
\text { disponibilidade e demanda do } \\
\text { serviço em cada região. }\end{array}$ \\
\hline & & $\begin{array}{c}\text { Apresentar e levar a aprovação, as } \\
\text { alterações aos setores } \\
\text { responsáveis. }\end{array}$ \\
\hline & $\begin{array}{l}\text { Definição das funções de } \\
\text { cada profissional. }\end{array}$ & $\begin{array}{c}\text { Organizar as atividades de cada } \\
\text { profissional. }\end{array}$ \\
\hline
\end{tabular}


Estabelecidas as operações e ações, a próxima etapa foi analisar a viabilidade e factibilidade das ações por meio da matriz do momento estratégico. A matriz do momento estratégico mostrou que o maior déficit encontrado foi a falta de poder de decisão entre os executores, pois, os recursos humanos e materiais para execução e manutenção se encontram em sua grande maioria disponíveis. Como estratégia de superação apontouse a sensibilização dos gestores.

As execuções dos apontamentos levantados do desenvolvimento do plano operativo iniciaram-se simultaneamente a conclusão do plano operativo. Operações e ações começaram a ser desenvolvidas e levadas aos gestores.

A finalização do plano operativo foi através do estabelecimento de indicadores de monitoramento e avaliação para o acompanhamento de sua execução, momento táticooperacional. Como indicadores das operações foram definidas verificações objetivas do cumprimento ou não do proposto, através da verificação de documentos legais, conforme mostra a TABELA 3.

TABELA 3 - MATRIZ DE INDICADORES DE AVALIAÇÃO

\begin{tabular}{c|c|c}
\hline OPERAÇÕES & INDICADORES & FONTES DE VERIFICAÇÃO \\
\hline $\begin{array}{c}\text { Elaboração e aprovação de } \\
\text { um projeto de coordenação } \\
\text { farmacêutica para o serviço }\end{array}$ & $\begin{array}{c}\text { Existência de uma } \\
\text { coordenação farmacêutica }\end{array}$ & $\begin{array}{c}\text { Organograma da secretaria } \\
\text { municipal de saúde }\end{array}$ \\
\hline $\begin{array}{c}\text { Promoção sobre a importância } \\
\text { da implantação de uma } \\
\text { coordenação farmacêutica } \\
\text { junto ao meio de controle }\end{array}$ & $\begin{array}{c}\text { Projeto de uma coordenação } \\
\text { farmacêutica no plano } \\
\text { municipal de saúde }\end{array}$ & Plano municipal de saúde \\
\hline $\begin{array}{c}\text { Revisão do projeto de } \\
\text { regionalização do serviço }\end{array}$ & $\begin{array}{c}\text { Alteração da regionalização } \\
\text { do serviço }\end{array}$ & Portaria publicada. \\
\hline $\begin{array}{c}\text { Identificação da necessidade } \\
\text { de recursos humanos }\end{array}$ & $\begin{array}{c}\text { Adequação do número de } \\
\text { farmacêuticos ao serviço }\end{array}$ & Recursos humanos \\
\hline $\begin{array}{c}\text { Definição das funções de cada } \\
\text { profissional }\end{array}$ & $\begin{array}{c}\text { Existência de um protocolo } \\
\text { de serviço farmacêutico }\end{array}$ & $\begin{array}{c}\text { Protocolos da Secretária } \\
\text { Municipal de Saúde }\end{array}$ \\
\hline
\end{tabular}




\section{DISCUSSÃO}

A AF enfrenta grandes desafios para a sua estruturação e implementação (OLIVEIRA; ASSIS; BARBONI, 2010). O plano operativo executado através do PES teve como foco uma farmácia básica. Entretanto, o problema relacionado à farmácia: pouca qualidade no atendimento ao usuário, apontou como causa convergente a ausência da AF no município. Esse resultado pode ser interpretado como forte evidência da importância da AF para a prestação dos serviços aos usuários do Sistema Único de Saúde.

O organograma da Secretária Municipal de Saúde do município não contempla um departamento e ou serviço de AF. Desse modo, percebe-se uma desarticulação das estruturas constantes ao ciclo da assistência, seleção, programação, aquisição, armazenamento, distribuição, e utilização. Como evidenciado através do momento explicativo. Tal situação acarreta vários problemas ao funcionamento das farmácias básicas do município, que carecem de planejamento, padronização do modo de trabalho, de estrutura física e de recursos humanos.

O momento explicativo, de onde foi extraída a causa convergente, foi realizado em conjunto com diferentes profissionais relacionados ao foco, mas sem a participação de representantes da gestão. Desse modo, pode-se perceber que a $\mathrm{AF}$ já se apresenta como uma necessidade aos profissionais relacionados com o serviço farmacêutico no município, diferentemente da pouca relevância mostrada pelos gestores.

Segundo Oliveira e colaboradores (2010), os desafios para a estruturação e a implementação de uma AF começam pela conscientização, por parte dos gestores da importância da estruturação da AF municipal. Durante o desenvolvimento do trabalho, o momento normativo apontou operações e ações no sentido de promover a conscientização dos gestores. Uma vez que AF se organiza como política de saúde, sua realização como imagem-objetivo, passou por discussões fora do âmbito local da farmácia.

A implantação integral da $\mathrm{AF}$ requer de recursos para a qualificação da gestão da mesma. A AF não pode ser entendida pelos gestores somente como a manutenção adequada de suprimentos, pois se os serviços não funcionam de forma satisfatória, alocar mais recursos em medicamentos implica aumentar as perdas, devido à ineficiência no gerenciamento dos serviços. (VIEIRA, FABIOLA SULPINO, 2010).

Resultados obtidos como a operação: elaborar e aprovar um projeto de coordenação farmacêutica para o serviço e promover a importância da implantação de uma coordenação farmacêutica junto ao meio de controle social, colaboram com esse 
apontamento. A dificuldade com os gestores também foi evidenciada no momento normativo, em que se apresentou como maior déficit.

Outro ponto que pode se relacionar a importância da implantação da AF no município, se refere à dificuldade encontrada na adesão dos participantes ao plano operativo. A ausência dos gestores na primeira oficina levou-se a um desmerecimento da prioridade do trabalho. Entretanto, essa dificuldade apresentada não influenciou o seu desenvolvimento nas demais oficinas.

\section{CONCLUSÃO}

O PES apresenta-se como uma ferramenta adequada ao gerenciamento de projetos, pois permite uma análise da situação real, definição de uma nova situação, construção da viabilidade do plano e monitoramento do plano.

Sua execução permite o desenvolvimento de várias habilidades e competências essenciais ao processo de gestão. Destaca-se como característica, o envolvimento de diferentes indivíduos (atores), uma vez que traz o aumento das perspectivas sobre o foco, além de possibilitar o empoderamento desses atores sobre transformações em suas realidades.

Segundo Dias e colaboradores, 2012, o PES é democrático ao processo de planejamento e gestão, ocorre através da valorização e interpretação de cada indivíduo sobre a realidade. As organizações públicas brasileiras, incluídas as da esfera da saúde, remetem à população e seus trabalhadores, sentimentos de perplexidades, impotência e sofrimento. Possibilita, através do entendimento da dinâmica social e do funcionamento das organizações, a quebra de paradigma.

Entretanto, há de se considerar dificuldades limitantes referentes à governabilidade, habilidade importante ao seu desenvolvimento. Compreende-se que o aprimoramento da competência governabilidade é um fator importante as etapas do método. A amplitude de certos problemas pode demandar recursos não disponíveis. De acordo com lida, 1993, nesse caso, a governabilidade é realizada através de demandas a atores que possuam tal governabilidade. Essa dificuldade pôde ser evidenciada claramente no desenvolvimento do plano operativo, em que as ações para implantação de uma AF no município, demandaram fortemente de recursos não disponíveis. 


\section{REFERÊNCIAS}

BARRETO, J. L.; GUIMARAES, M. do C. L. Avaliação da gestão descentralizada da assistência farmacêutica básica em municípios baianos, Brasil. Cad. Saúde Pública, Rio de Janeiro, v. 26, n. 6, p. 1207-1220, 2010.

BRASIL. Lei 8.080, de 19 de setembro de 1990. Dispoe sobre as Condições para a Promoção, Proteção e Recuperação da Saúde, a Organização e Funcionamento dos Serviços Correspondentes e dá outras Providências. Diário Oficial da União, Brasília-DF, 1990.

BRASIL, Portaria no 3916, de 30 de outubro de 1998. Aprova a Política Nacional de Medicamentos. Diário Oficial da União, Brasília-DF, 1998.

BRASIL, Resolução CNS no 338, de 06 de maio de 2004. Aprova a Política Nacional de Assistência Farmacêutica. Diário Oficial da União. Brasília,-DF, 2004

DIAS, R. C. et al. Impacto do Planejamento Estratégico Situacional em um Ambulatório de Atenção Especializada. Revista de Gestão em Sistemas de Saúde, v. 01, n. 01, p. 83-96, 2012.

IIDA, I. Planejamento estratégico situacional. Produção, v. 3, n. 2, p. 113-125, dez. 1993.

MATUS, C. Política, planejamento e governo. Brasília: Instituto de Pesquisa Econômica Aplicada, 1993.

OLIVEIRA, L. C. F. De; ASSIS, M. M. A.; BARBONI, A. R. Assistência Farmacêutica no Sistema Único de Saúde: da Política Nacional de Medicamentos à Atenção Básica à Saúde. Ciência \& Saúde Coletiva, v. 15, p. 3561-3567, nov. 2010.

VIEIRA, F. S. Assistência farmacêutica no sistema público de saúde no Brasil. Revista Panamericana de Salud Pública, v. 27, n. 2, p. 149-156, fev. 2010.

VIEIRA, F. S. Avanços e desafios do planejamento no Sistema Único de Saúde. Ciência \& Saúde Coletiva, v. 14, p. 1565-1577, out. 2009. 